\title{
Secular changes and predictors of adult height for 86105 male and female members of the Thai Cohort Study born between 1940 and 1990
}

\author{
Susan Jordan, ${ }^{1}$ Lynette Lim, ${ }^{2}$ Sam-ang Seubsman, ${ }^{3}$ Christopher Bain, ${ }^{1}$ \\ Adrian Sleigh, ${ }^{2}$ the Thai Cohort Study Team
}

${ }^{1}$ School of Population Health, University of Queensland, Brisbane, Australia ${ }^{2}$ National Centre for Epidemiology and Population Health, ANU College of Medicine and Health Sciences, the Australian National University, Canberra, Australia ${ }^{3}$ Thai Health-Risk Transition Study, School of Human Ecology, Sukhothai, Thammathirat Open University, Nonthaburi, Thailand

\section{Correspondence to}

Dr Susan Jordan, School of Population Health, the University of Queensland, Public Health Building, Herston Road, Herston Old 4006, Australia; s.jordan@uq.edu.au

The Thai Cohort Study team consists of: Thailand: Jaruwan Chokhanapitak, Chaiyun

Churewong, Suttanit Hounthasarn, Suwanee Khamman, Daoruang Pandee, Suttinan Pangsap, Tippawan Prapamontol, Janya Puengson, Yodyiam Sangrattanakul, Sam-ang Seubsman, Boonchai Somboonsook, Nintita Sripaiboonkii, Pathumvadee Somsamai, Duangkae Vilainerun, Wanee

Wimonwattanaphan.Australia: Chris Bain, Emily Banks, Cathy Banwell, Bruce Caldwell, Gordon Carmichael, Tarie Dellora, Jane Dixon, Sharon Friel, David Harley, Matthew Kelly, Tord Kjellstrom, Lynette Lim, Anthony McMichael, Tanya Mark, Adrian Sleigh, Lyndall Strazdins.

Accepted 6 July 2010 Published Online First 30 August 2010

\section{UNLOCKED}

This paper is freely available online under the BMJ Journals unlocked scheme, see http:// jech.bmi.com/site/about/ unlocked.xhtml

\begin{abstract}
Background Height trends can be useful indicators of population health but, despite Thailand's rapid socioeconomic development since the 1950s, few studies have examined accompanying secular changes in adult height or the effects of the transition on the heights of rural versus urban populations. This study therefore sought to document average heights in different age groups of rural and urban Thais and to investigate factors associated with attained height.
\end{abstract}

Methods Data from 86105 Thai Cohort Study participants was used to estimate mean heights for men and women in different birth year groups. Simple regression was used to calculate the change in height per decade of birth year among those based in rural or urban locations as children. Multiple linear regression was used to investigate effects of other childhood factors on height.

Results Overall, average heights were found to have increased by approximately $1 \mathrm{~cm}$ per decade in those born between 1940 and 1990. However, the rate of increase was $0.4-0.5 \mathrm{~cm}$ per decade greater among urban-based Thais compared with those from the countryside. Parental education levels, household assets, birth size, sibling number, birth rank and region of residence were also significantly associated with adult height.

Conclusions These data suggest a marked secular increase in Thai heights in the second half of the 20th century probably reflecting improved childhood health and nutrition over this time. Rural-born Thais, who benefited to a lesser extent from the changes, may face future health challenges with greater risks of, among other things, obesity and its health consequences.

Thailand's relatively rapid transition from traditional subsistence to modern consumer economy has already produced a demographic transition in death rates ${ }^{1}$ and, in the 20th century, much of the dramatic decline in these rates was due to falling infant and child mortality. ${ }^{2}$ As attained adult height reflects, among other things, childhood health and nutrition, alongside falling infant and childhood mortality, it would be expected that marked secular increases in height have occurred among Thais over the past 50 years.

As well as reflecting childhood conditions, attained adult height is associated with mortality in later life. Overall, short stature is associated with a higher total mortality, ${ }^{3-6}$ although associations with cause-specific mortality appear to vary such that most studies have found an inverse association between attained adult height and coronary heart disease and stroke, ${ }^{4} 78$ but a positive association with cancers such as those of the breast, prostate and colon. ${ }^{10}$ The documentation of trends in height thus can not only help chart improvements in population health, but may also be a useful indicator of future health burdens.

Despite being well along its health transition, little information on secular changes in Thai height has been published. ${ }^{11} 12$ Furthermore, despite distinct income inequalities that have existed between rural and urban Thais, ${ }^{13}$ height changes in the two groups have not been compared. For these reasons the aims of the current study are to: document the average attained heights in different age groups of rural and urban-based participants in a large cohort study of male and female open university students in Thailand (the Thai Cohort Study), and to investigate factors associated with these secular changes.

\section{METHODS}

The overall aim of the Thai Cohort Study is to examine the health consequences of Thailand's rapid socioeconomic development and environmental change. ${ }^{1}$ To this end participants were recruited from the student body of the Sukhothai Thammathirat Open University (STOU). STOU students reside all over Thailand and have a modest socioeconomic status. Many are rural dwellers and most have full-time jobs. In 2005, all STOU students who had completed at least their first semester of study (approximately 200000) were mailed a questionnaire, information sheet and consent form. A total of 87134 students (47314 women and 39820 men) returned completed questionnaires (44\%). Participants were asked to record their height in centimetres $(\mathrm{cm})$. We also requested information on factors related to socioeconomic status during childhood, including the area (rural or urban) in which they resided as a child (aged 10-12 years). The sensitive period for socioeconomic influences on height may be earlier than age 10-12 years, but this age was specified for greater accuracy of recall.

\section{Statistical analyses}

Analyses were conducted separately for men and women. As we were interested in comparing heights across different age groups, participants were initially grouped by birth year. Participants' ages ranged from 15 to 87 years, so we excluded 
those aged over 65 years $(n=76)$ to avoid a long tail in the first birth year category. Mean heights (cm) with 95\% CI were calculated for each group. A similar comparison was made stratifying by area of residence (rural/urban) at age 10-12 years. The data were further assessed using simple linear regression with height as the outcome and mean-centred birth year (in decades) as the predictor. Stratification of these models by childhood area of residence suggested effect modification, so an interaction term for birth year and area of residence was added to the original model. Other childhood factors associated with attained adult height were assessed using multiple linear regression. All variables related to childhood circumstance were entered into the model, including: attained maternal and paternal level of education; sibling number; birth rank; birth size; whether breast fed in infancy; and the numbers of material resources in the home such as electricity, radio, television or a refrigerator at age 10-12 years. Variables relating to current circumstances (such as current income) were excluded to minimise the possibility of reverse causation.

We had two possible measures of birth size: self-reported birth weight (g) and relative birth size recalled by relatives (small, normal, large). When both questions were answered, the two measures were highly correlated, but the data for both were missing for a large proportion of participants. More $(75 \%$ vs $40 \%$ ) responded to the question on birth size recalled by relatives than on birth weight, so we used the former to estimate the effect on height change. Because a large amount of birth size data was missing we have reported the estimates for other childhood factors unadjusted for birth size. Of note, the only estimate materially changed by the addition of birth size to the model was that for breast feeding.

Birth rank was initially analysed in strata of number of siblings and age groups. The results from stratified analyses were similar to unstratified analyses and suggested that those later born were more likely to be taller than those first born, thus only a term for first born versus later born was included in the final model.

A variable indicative of childhood material resources was created by summing the positive responses from the following question: "Which of the following did your home have when you were 10-12 years old? Electricity (generator or outside line), microwave, refrigerator, electric fan, air conditioning, television, video/tape/CD player, computer, telephone, mobile telephone, water heater, or washing machine.'

Ethics approval was obtained from Sukhothai Thammathirat Open University Research and Development Institute (protocol 0522/10) and the Australian National University Human Research Ethics Committee (protocol 2004344). Informed written consent was obtained from all participants.

\section{RESULTS}

Overall, 86105 participants (99\% of all cohort members) aged under 66 years reported their height. Table 1 shows the frequencies of various demographic factors as well as the indicators of childhood circumstances.

We found among both female and male participants that height increased with later birth year (table 2).

For women, those born between 1986 and 1990 were almost $3 \mathrm{~cm}$ taller than women born between 1940 and 1956. Similarly, men born between 1986 and 1990 were on average $4 \mathrm{~cm}$ taller than men born between 1940 and 1956. This difference was even greater when considered jointly with area of residence at age 10-12 years (table 3 ): the youngest group of urban-based men was on average $6.4 \mathrm{~cm}$ taller than the oldest group of rural-based men and the equivalent difference for women was $4.3 \mathrm{~cm}$.

Mean heights with $95 \%$ CI for men (figure $1 \mathrm{~A}$ ) and women (figure 1B) by rural/urban location at age 10-12 years are shown in figure 1. Linear regression suggested an overall increase in height of $1.50 \mathrm{~cm}$ (95\% CI 1.36 to 1.63$)$ per decade for male urban dwellers compared with $1.01 \mathrm{~cm}$ (95\% CI 0.93 to 1.09) per decade for male rural dwellers. For women the estimates were $1.32 \mathrm{~cm}$ (95\% CI 1.20 to 1.43 ) for urban dwellers and $0.91 \mathrm{~cm}$ (95\% CI 0.82 to 0.99 ) for rural dwellers. The interaction terms for birth year and area of residence (rural/urban) aged $10-12$ years were highly significant for both men and women $(\mathrm{p}<0.0001)$.

Table 4 shows the estimates of effect on height change of various measures of childhood circumstances. Participants' birth year remained a significant predictor of height after adjustment for the measured childhood factors, with approximately half a centimetre increase in height per decade for both women and men.

Several other childhood factors were significantly associated with attained adult height. Educational attainment of both parents was related to height of both men and women. Maternal education level was more important for women, such that those with mothers with tertiary education were on average $1.1 \mathrm{~cm}$ (95\% CI 0.7 to $1.5 \mathrm{~cm}$ ) taller than those whose mothers had no formal education; for men, paternal level of education was associated with the larger effect, such that men whose fathers had tertiary education were on average $1.4 \mathrm{~cm}$ (95\% CI 1.0 to $1.8 \mathrm{~cm}$ ) taller than men whose fathers had no formal education. Of the factors considered, birth size (recalled by relatives) was associated with the largest effects on height for both sexes. Women who were considered large or overweight at birth were on average approximately $2.8 \mathrm{~cm}$ taller than those considered small or underweight at birth, and the corresponding estimate for men was $3.3 \mathrm{~cm}$. Those who reported having been breast fed were on average approximately $0.3 \mathrm{~cm}$ taller than those who reported they were not breast fed, although this effect disappeared when birth size was added to the model $(0.05 \mathrm{~cm}$; $95 \%$ CI -0.18 to 0.27 for women and $-0.01 \mathrm{~cm} ; 95 \% \mathrm{CI}-0.33$ to 0.31 for men). Having larger numbers of siblings was associated with shorter stature, with a more pronounced effect in men than women. Birth order was also important with both later born men and women tending to be taller than those first born.

In both sexes, those with a larger number of assets in the home at age 10-12 years were on average taller than those who had few assets, although the effect was not as large in women. As some of the technologies included in the assets score did not exist when the older participants were children, in addition to adjusting for birth year, we also stratified by birth year group The same pattern was evident for all groups (results not shown) so we have presented only the birth year adjusted estimates.

\section{DISCUSSION}

Using data from 86105 Thai open university students we have found evidence of a substantial increase in average attained adult height among both men and women born between 1940 and 1990, amounting overall to approximately $1 \mathrm{~cm}$ increase in height per decade. A rural/urban difference was strikingly evident such that the rate of increase in height has been approximately $0.5 \mathrm{~cm}$ per decade greater among men reared in urban areas compared with those from rural areas and $0.4 \mathrm{~cm}$ per decade greater for urban compared with rural women. Multivariable analyses showed that other indicators of childhood 
Table 1 Frequencies of demographic and childhood characteristics for female and male Thai Cohort Study participants with recorded height

\begin{tabular}{|c|c|c|c|c|}
\hline & \multicolumn{2}{|c|}{ Women $N=47207$} & \multicolumn{2}{|c|}{ Men $\mathrm{N}=\mathbf{3 8 8 9 8}$} \\
\hline & $\overline{\mathbf{N}^{*}}$ & $\%$ & $\overline{\mathbf{N}^{*}}$ & $\%$ \\
\hline \multicolumn{5}{|l|}{ Birth year } \\
\hline $1940-55$ & 584 & 1.24 & 1412 & 3.63 \\
\hline $1956-65$ & 4401 & 9.32 & 6336 & 16.29 \\
\hline $1966-75$ & 13438 & 28.47 & 13565 & 34.87 \\
\hline $1976-85$ & 27148 & 57.51 & 16746 & 43.05 \\
\hline $1986-90$ & 1636 & 3.47 & 839 & 2.16 \\
\hline \multicolumn{5}{|l|}{ Current area of residence } \\
\hline Rural & 22041 & 47.01 & 19211 & 49.76 \\
\hline Urban & 24842 & 52.99 & 19399 & 50.24 \\
\hline \multicolumn{5}{|l|}{ Current region of residence } \\
\hline Bangkok & 9058 & 19.32 & 5618 & 14.57 \\
\hline Central & 12187 & 26.00 & 8740 & 22.67 \\
\hline North & 8021 & 17.11 & 7556 & 19.60 \\
\hline Northeast & 8410 & 17.94 & 9411 & 24.41 \\
\hline East & 2909 & 6.21 & 2364 & 6.13 \\
\hline South & 6290 & 13.42 & 4860 & 12.61 \\
\hline \multicolumn{5}{|c|}{ Current monthly income (Baht) } \\
\hline$\leq 3000$ & 4842 & 10.52 & 4412 & 11.63 \\
\hline $3001-7000$ & 16976 & 36.88 & 9000 & 23.72 \\
\hline $7001-10000$ & 10887 & 23.65 & 8735 & 23.02 \\
\hline $10001-20000$ & 9551 & 20.75 & 9551 & 20.75 \\
\hline $20001-30000$ & 2325 & 5.05 & 2900 & 7.64 \\
\hline$>30000$ & 1446 & 3.14 & 2142 & 5.64 \\
\hline \multicolumn{5}{|l|}{ Level of education } \\
\hline Junior high school & 1054 & 2.24 & 1919 & 4.95 \\
\hline High school & 19416 & 41.24 & 19473 & 50.18 \\
\hline Diploma/certificate & 14449 & 30.69 & 8801 & 22.68 \\
\hline University degree & 12156 & 25.82 & 8611 & 22.19 \\
\hline \multicolumn{5}{|c|}{ Father's levels of education } \\
\hline No formal education & 2353 & 5.07 & 2432 & 6.37 \\
\hline Primary level & 28158 & 60.72 & 23613 & 61.84 \\
\hline Secondary level & 9249 & 19.94 & 7031 & 18.41 \\
\hline Tertiary level & 3958 & 8.54 & 3085 & 8.08 \\
\hline Do not know & 2655 & 5.73 & 2021 & 5.29 \\
\hline \multicolumn{5}{|c|}{ Mother's level of education } \\
\hline No formal education & 4433 & 9.48 & 4258 & 11.08 \\
\hline Primary level & 33583 & 71.81 & 27608 & 71.83 \\
\hline Secondary level & 4510 & 9.64 & 3146 & 8.18 \\
\hline Tertiary level & 2544 & 5.44 & 1839 & 4.78 \\
\hline Do not know & 1697 & 3.63 & 1586 & 4.13 \\
\hline \multicolumn{5}{|l|}{ Number of siblings } \\
\hline 0 & 2218 & 4.76 & 1361 & 3.54 \\
\hline 1 & 11375 & 24.41 & 7709 & 20.04 \\
\hline 2 & 11754 & 25.22 & 8517 & 22.14 \\
\hline 3 & 7419 & 15.92 & 6099 & 15.86 \\
\hline 4 & 4621 & 9.92 & 4359 & 11.33 \\
\hline $5+$ & 9216 & 19.78 & 10416 & 27.08 \\
\hline \multicolumn{5}{|c|}{ Birth size recalled by relatives } \\
\hline Small or underweight & 9231 & 19.79 & 6285 & 16.41 \\
\hline Normal & 22201 & 47.61 & 18171 & 47.45 \\
\hline Large or overweight & 4454 & 9.55 & 3278 & 8.56 \\
\hline Do not know & 10747 & 23.05 & 10558 & 27.57 \\
\hline \multicolumn{5}{|l|}{ Breast fed } \\
\hline Yes & 41141 & 91.62 & 34453 & 94.07 \\
\hline No & 3762 & 8.38 & 2170 & 5.93 \\
\hline \multicolumn{5}{|c|}{ Area of residence age $10-12$ years } \\
\hline Rural & 34711 & 74.16 & 29843 & 77.65 \\
\hline Urban & 12095 & 25.84 & 8589 & 22.35 \\
\hline \multicolumn{5}{|c|}{ No of household assets when aged $10-12$ years } \\
\hline $0-1$ & 6369 & 13.56 & 9344 & 24.18 \\
\hline
\end{tabular}

Table 1 Continued

\begin{tabular}{|c|c|c|c|c|}
\hline & \multicolumn{2}{|c|}{ Women $N=47207$} & \multicolumn{2}{|c|}{ Men N=38898 } \\
\hline & $\mathbf{N}^{*}$ & $\%$ & $\mathbf{N}^{*}$ & $\%$ \\
\hline $2-4$ & 17649 & 37.57 & 14090 & 36.46 \\
\hline $5-6$ & 15574 & 33.16 & 10640 & 27.53 \\
\hline$>6$ & 7379 & 15.71 & 4574 & 11.84 \\
\hline
\end{tabular}

circumstances, such as parental education levels, household assets, birth size, sibling number and birth rank, were also significantly associated with attained adult height but did not completely account for the effect of birth year.

The strengths of our study include the large numbers of both male and female participants. The study group represents well the overall Thai population in terms of sex distribution, median income, region of residence and religion. ${ }^{1}$ However, individuals in their 20s and 30s were overrepresented in the study, and it is also likely that the study population includes fewer individuals of very high or very low socioeconomic status. Study participants are also slightly more educated than the average Thai. Our results may thus be most applicable to the group of upwardly mobile Thais who have been most affected by the socioeconomic transition of Thailand over the past 50 years.

One limitation of the study was that height was self-reported and overestimation is the norm in this situation including among STOU students,. ${ }^{14}$ Another limitation was the crosssectional nature of the data collection, such that height was not recorded at the same age for all participants. Some younger participants would not have reached full adult height at the time of the survey, so we may have underestimated the mean adult height for those born between 1986 and 1990. Furthermore, if height is associated with survival in this population (as it appears to be in others) then it may be that the older participants here are, on average, taller than their Thai birth cohort as a whole. It is possible, therefore, that the height differences between older and younger birth cohorts are actually greater than we have found here. Conversely, age-related shrinkage might mean that the recorded heights of our older cohorts are lower than their attained adult heights. However, we feel this is less likely to be a problem because height was self-reported. Studies (from western populations) suggest that overreporting of height significantly increases with age, ${ }^{15}{ }^{16}$ probably reflecting a tendency among older people to report attained rather than current height.

Some of the factors we investigated related to childhood circumstances present many years earlier, so there may have been recall errors. However, it is unlikely that taller participants would recall events in a systematically different way to shorter participants and thus bias our results. We also did not have any information on parental or sibling heights so were unable to consider the role of genetic factors in attained adult height.

Table 2 Mean height in centimetres for women and men in the Thai Cohort Study by birth year group

\begin{tabular}{|c|c|c|c|c|}
\hline \multirow[b]{2}{*}{ Birth year } & \multicolumn{2}{|l|}{ Women } & \multicolumn{2}{|l|}{ Men } \\
\hline & $\begin{array}{l}\text { Mean } \\
\text { height (SD) }\end{array}$ & $\mathbf{N}$ & $\begin{array}{l}\text { Mean } \\
\text { height (SD) }\end{array}$ & $\mathbf{N}$ \\
\hline $1940-55$ & $156.2(5.5)$ & 584 & $166.3(6.0)$ & 1413 \\
\hline $1956-65$ & $156.4(5.3)$ & 4401 & $167.3(5.8)$ & 6336 \\
\hline $1966-75$ & $157.2(5.4)$ & 13438 & $168.0(5.7)$ & 13565 \\
\hline $1976-85$ & $158.2(5.6)$ & 27148 & $169.2(6.1)$ & 16746 \\
\hline $1986-90$ & $159(5.9)$ & 1636 & $170.3(6.3)$ & 839 \\
\hline
\end{tabular}


Table 3 Mean height in cms (SD) by age group for men and women according to whether they lived in an urban/rural area aged 10-12 years

\begin{tabular}{llllll}
\hline \multirow{2}{*}{ Birth year } & Women & & & Men \\
\cline { 2 - 3 } \cline { 6 - 7 } & Rural & Urban & & Rural & Urban \\
\hline $1940-55$ & $156.0(5.4)$ & $156.5(5.5)$ & & $166.0(6.1)$ & $166.9(5.8)$ \\
$1956-65$ & $156.3(5.4)$ & $156.7(5.3)$ & & $166.9(5.8)$ & $168.5(5.7)$ \\
$1966-75$ & $156.9(5.4)$ & $157.9(5.6)$ & & $167.6(5.7)$ & $169.5(5.8)$ \\
$1976-85$ & $157.8(5.6)$ & $159.3(5.7)$ & & $168.7(6.0)$ & $171.2(6.0)$ \\
$1986-90$ & $158.5(5.7)$ & $160.3(6.2)$ & & $169.6(6.2)$ & $172.4(6.1)$ \\
\hline
\end{tabular}

To our knowledge only one study has previously published data on secular changes in attained adult height in Thailand. ${ }^{11}$ That study used the measured heights of male, Bangkok-based
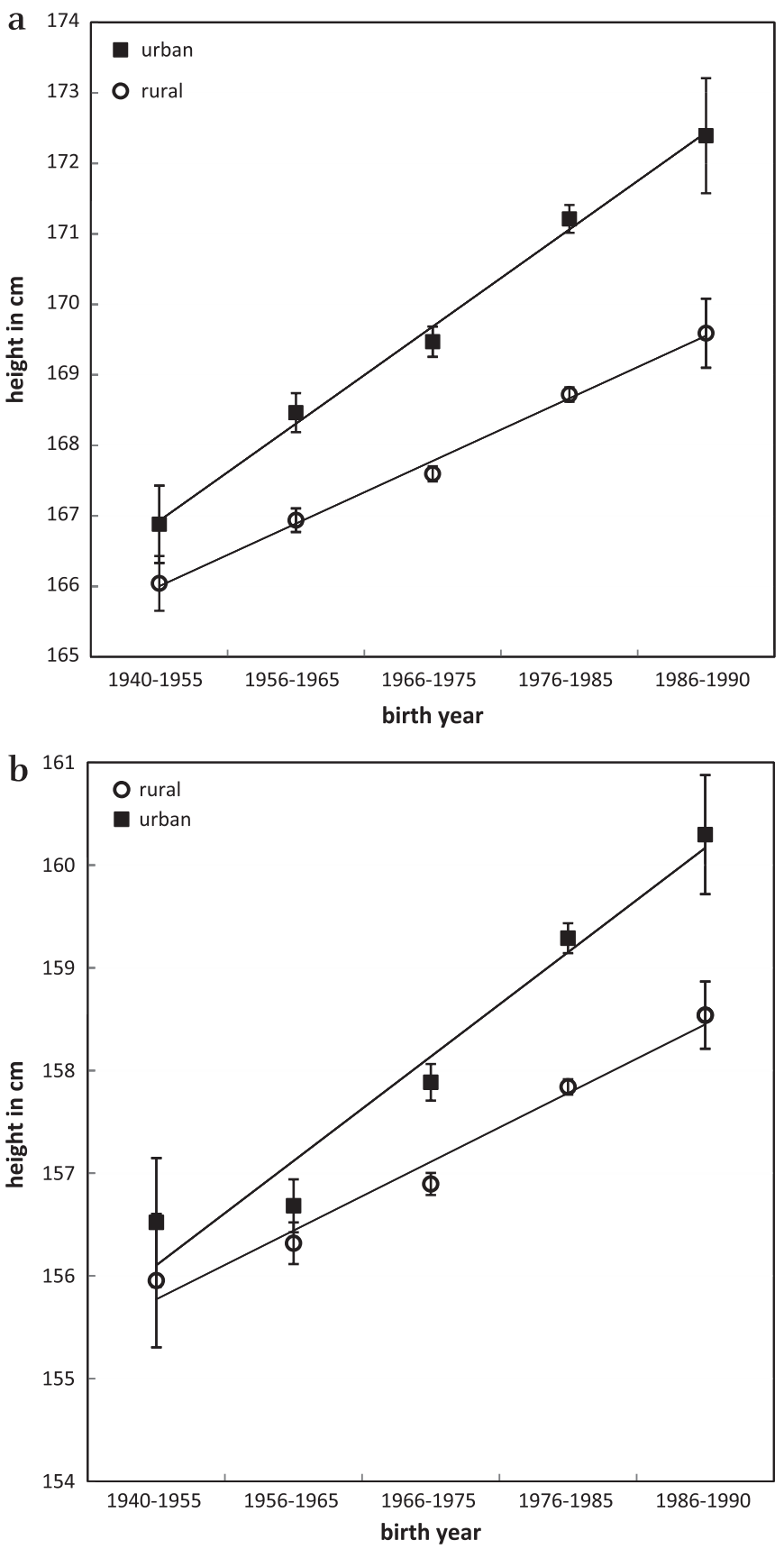

Figure 1 Mean heights and $95 \% \mathrm{Cl}$ among (a) Thai men and (b) Thai women who resided in rural or urban locations at age $10-12$ years. military recruits born between 1951 and 1985, and found that average height increased by approximately $1.3 \mathrm{~cm}$ per decade. Urban/rural differences could not be considered in that study and the population may have been of slightly lower socioeconomic status, but the findings overall were in close accord with what we have found here for men. Studies of urban-based populations from China ${ }^{17}$ and Hong $\mathrm{Kong}^{18}$ covering similar time periods suggested similar secular increases of $1.3 / 1.1 \mathrm{~cm}$ per decade and $1.2 / 0.9 \mathrm{~cm}$ per decade, respectively, for men and women. For Japan ${ }^{19}$ and Korea ${ }^{20}$ data from longer time periods have suggested slightly greater increases of $1.5 \mathrm{~cm}$ (Japan national) and $2 \mathrm{~cm}$ (Korea urban) per decade for women and $2 \mathrm{~cm}$ per decade for men (Japan).

Attained adult height reflects, among other things, childhood health and nutrition, ${ }^{21} 22$ and thus the greater average heights that we have observed in the younger compared to the older participants suggests a marked improvement in childhood conditions in Thailand over the second half of the 20th century. This is in keeping with the large increase in gross domestic product per capita that has occurred over this period (gross domestic product per capita increased from US\$990 in 1950 to US\$4267 in 1990; international purchasing power parity dollars-constant year 2000 prices). ${ }^{23}$ However, the benefit seems to have been unequally distributed as the rate of increase in height appears to have been substantially greater for urban compared with rural dwellers. Rural-urban differences in height have been reported in European ${ }^{24} 25$ and Asian ${ }^{26} 27$ populations but, at least in the European studies, height differences by locale appear to be decreasing rather than increasing. However, in Thailand increasing income inequality between rural and urban populations has been apparent from the 1960s through to at least the early 1990 s, ${ }^{13}$ spanning the years in which the majority of our cohort members were children. The increasing height differences we have observed between rural and urban dwellers therefore probably reflects widening economic disparities between urban and rural communities and concomitant effects on childhood health and nutrition.

Our other findings in relation to childhood circumstances reflect what has been observed in a variety of countries (both developed and developing), including the positive relation between height and birth size ${ }^{27-29}$ parental levels of education, ${ }^{25}$ and number of assets in the family home ${ }^{30}$; and the inverse relation between height and family size. ${ }^{28} 3132$ The suggestion that later born children are on average taller than those first born has also been reported elsewhere, ${ }^{33} 34$ although others have found the inverse to be true. ${ }^{28} 35$ It may be that issues such as birth spacing and cultural influences on care patterns in families account for these differences, but we did not have the relevant data to explore this issue further. Overall, our findings in relation to childhood circumstances most likely reflect the effect on growth of access to resources in early childhood and ultimately the adequacy of nutrition (both quality and quantity) and the ability to control childhood infection. ${ }^{21} 22$

Our findings may also have implications for future health trends in Thailand, particularly in relation to chronic diseases. In general, greater height is associated with lower overall mortality. $^{3-6}$ In particular, greater height is associated with a decreased risk of death from stroke, ${ }^{4-6}$ coronary artery disease, ${ }^{4} 7$ respiratory disease ${ }^{4-6}$ and external causes. ${ }^{6}$ This suggests that the secular increase in height should have beneficial effects on the profile of some chronic diseases in Thailand. A caveat to this is that the prevalence of other risk factors for diseases of the circulatory system is likely to increase as western diets and 
Table 4 Adjusted* estimates of height change $(\mathrm{cm})$ for a unit change in factors associated with childhood circumstances among Thais

\begin{tabular}{|c|c|c|c|c|c|c|}
\hline \multirow[b]{2}{*}{ Factor } & \multicolumn{3}{|l|}{ Women } & \multicolumn{3}{|l|}{ Men } \\
\hline & Estimate $\mathbf{c m}$ & $95 \% \mathrm{Cl}$ & p Value & Estimate cm & $95 \% \mathrm{CI}$ & p Value \\
\hline Per decade increase in birth year & 0.67 & 0.58 to 0.76 & $<0.0001$ & 0.58 & 0.49 to 0.67 & $<0.0001$ \\
\hline None & Reference & & & Reference & & \\
\hline Primary & 0.54 & 0.33 to 0.75 & $<0.0001$ & 0.26 & 0.03 to 0.49 & 0.03 \\
\hline Secondary & 0.74 & 0.46 to 1.02 & $<0.0001$ & 0.63 & 0.29 to 0.96 & 0.0002 \\
\hline None & Reference & & & Reference & & \\
\hline Primary & 0.41 & 0.14 to 0.68 & 0.003 & 0.50 & 0.20 to 0.79 & 0.003 \\
\hline Secondary & 0.55 & 0.26 to 0.84 & 0.0002 & 0.96 & 0.64 to 1.29 & $<0.0001$ \\
\hline Tertiary & 0.65 & 0.29 to 1.01 & 0.0004 & 1.43 & 1.02 to 1.84 & $<0.0001$ \\
\hline \multicolumn{7}{|l|}{ Birth size as recalled by relatives $\dagger$} \\
\hline Small or underweight & Reference & & & Reference & & \\
\hline Yes & 0.30 & 0.10 to 0.51 & 0.004 & 0.27 & 0.007 to 0.55 & 0.06 \\
\hline \multicolumn{7}{|l|}{ No of siblings } \\
\hline 0 & Reference & & & Reference & & \\
\hline 1 & -0.08 & -0.37 to 0.20 & 0.55 & -0.36 & -0.74 to 0.03 & 0.07 \\
\hline 2 & -0.27 & -0.56 to 0.02 & 0.07 & -0.48 & -0.87 to -0.09 & 0.01 \\
\hline 3 & -0.39 & -0.70 to -0.09 & 0.01 & -0.75 & -1.16 to -0.34 & 0.0003 \\
\hline 4 & -0.70 & -1.03 to -0.37 & $<0.0001$ & -0.84 & -1.26 to -0.41 & 0.0001 \\
\hline $5+$ & -0.63 & -0.95 to -0.31 & $<0.0001$ & -1.19 & -1.60 to -0.78 & $<0.0001$ \\
\hline \multicolumn{7}{|l|}{ Birth order } \\
\hline First born & Reference & & & Reference & & \\
\hline Later born & 0.37 & 0.24 to 0.49 & $<0.0001$ & 0.31 & 0.16 to 0.46 & $<0.0001$ \\
\hline \multicolumn{7}{|c|}{ Area of residence when aged $10-12$ years } \\
\hline \multicolumn{7}{|c|}{ Assets in the home when aged $10-12$ years } \\
\hline
\end{tabular}

*Each factor adjusted for all others listed in the table apart from birth size recalled by relatives.

tEstimates calculated using data from those who reported birth size ( $75 \%$ of cohort), adjusted for all listed factors.

$\neq$ When birth size is added to the model the effect of breast feeding is negligible $(0.05 \mathrm{~cm}, 95 \% \mathrm{Cl}-0.18$ to 0.27 for women and $-0.01 \mathrm{~cm}, 95 \% \mathrm{Cl}-0.33$ to 0.31 for men).

lifestyles are increasingly adopted. Our work suggests that this could be of particular concern in rural areas where the relative height deficit combined with an increasingly energy-dense diet and sedentary lifestyle could result in substantial levels of obesity. Recent work based on the Thai National Health Examination Surveys suggest indeed that rates of obesity are increasing more rapidly in rural than urban areas. ${ }^{36}$ Height is also positively associated with a number of cancers, particularly those of the breast, prostate and colon. ${ }^{9}$ It is likely, therefore, that rates of these types of cancers (currently relatively rare in

\section{What is already known on this subject}

Thailand has undergone rapid socioeconomic development over the past 50 years with associate changes in population health. Secular trends in adult height reveal population health transitions, but such changes in Thailand, particularly urban/rural contrasts, are little studied.
Thailand compared with western populations) ${ }^{37}$ will increase with the increase in average heights.

In conclusion, we have found evidence of a marked secular increase in the height of Thais in the second half of the 20th century reflecting the rapid socioeconomic development of Thailand over this period. While these changes suggest overall

\section{What this study adds}

Average heights increased approximately $1 \mathrm{~cm}$ per decade in those born between 1940 and 1990, with urban Thais increasing approximately $0.5 \mathrm{~cm}$ more per decade than rural dwellers. Parental education, household assets, birth size, family size and birth rank were also significantly associated with height. This marked secular increase in Thai heights reflects improved child health and nutrition, but rural-born Thais have benefited less. They still tend to be of modest stature as adults, and now face a nutrition transition with a food surplus, obesity and its consequences. 
positive health outcomes, they may also reveal opportunities for health interventions, particularly for rural-born Thais who appear to have, at least until the latter part of the 20th century, benefited to a lesser extent from the secular trend in height.

Acknowledgements The authors would like to thank the staff at Sukhothai Thammathirat Open University (STOU) who assisted with student contact and the STOU students who are participating in the cohort study. They also thank Dr Bandit Thinkamrop and his team from Khon Kaen University for guiding them successfully through the complex data processing.

Funding This study was supported by the International Collaborative Research Grants Scheme with joint grants from the Wellcome Trust UK (WT071587MA) and the Australian National Health and Medical Research Council (NHMRC) (268055). SJ is supported by a Postdoctoral Public Health Training Fellowship from the Australian NHMRC.

\section{Competing interests None.}

Patient consent Obtained

Ethics approval Ethics approval was obtained from Sukhothai Thammathirat Open University Research and Development Institute (protocol 0522/10) and the Australian National University Human Research Ethics Committee (protocol 2004344).

Provenance and peer review Not commissioned; externally peer reviewed.

\section{REFERENCES}

1. Sleigh AC, Seubsman SA, Bain C. Cohort profile: the Thai Cohort of 87,134 Open University students. Int J Epidemiol 2008;37:266-72.

2. Data finder under 5 mortality rate. The World Bank, 2009. http://datafinder worldbank.org/under-5-mortality-rate (accessed 1 Dec 2009).

3. Jousilahti $\mathbf{P}$, Tuomilehto J, Vartiainen $\mathrm{E}$, et al. Relation of adult height to causespecific and total mortality: a prospective follow-up study of 31,199 middle-aged men and women in Finland. Am J Epidemiol 2000:151:1112-20.

4. Davey Smith G, Hart C, Upton M, et al. Height and risk of death among men and women: aetiological implications of associations with cardiorespiratory disease and cancer mortality. J Epidemiol Community Health 2000:54:97-103.

5. Song YM, Smith GD, Sung J. Adult height and cause-specific mortality: a large prospective study of South Korean men. Am J Epidemiol 2003;158:479-85.

6. Song YM, Sung J. Adult height and the risk of mortality in South Korean women. Am J Epidemiol 2008;168:497-505.

7. Williams SR, Jones E, Bell W, et al. Body habitus and coronary heart disease in men. A review with reference to methods of body habitus assessment. Eur Heart $J$ 1997:18:376-93

8. Silventoinen K, Magnusson PK, Tynelius P, et al. Association of body size and muscle strength with incidence of coronary heart disease and cerebrovascular diseases: a population-based cohort study of one million Swedish men. Int J Epidemiol 2009;38:110-18.

9. Gunnell D, Okasha M, Smith GD, et al. Height, leg length, and cancer risk: a systematic review. Epidemiol Rev 2001;23:313-42.

10. Sung J, Song YM, Lawlor DA, et al. Height and site-specific cancer risk: a cohor study of a Korean adult population. Am J Epidemiol 2009;170:53-64.

11. Seubsman SA, Sleigh AC. Change in mean height of Thai military recruits from 1972 through 2006. J Epidemiol 2009:19:196-201.

12. Sungthong R, Mo-suwan L, Chongsuvivatwong V, et al. Secular increases in weight, height and body mass index among school children of Hat Yai, Thailand: a 5 years follow-up study. Southeast Asian J Trop Med Public Health 1999;30:532-8.
13. Ikemoto Y, Uehara M. Income Inequality and Kuznets' Hypothesis in Thailand. Asian Econ J 2000;14:421-43.

14. Lim LL, Seubsman SA, Sleigh A. Validity of self-reported weight, height, and body mass index among university students in Thailand: implications for population studies of obesity in developing countries. Popul Health Metr 2009; 7:15

15. Kuczmarski MF, Kuczmarski RJ, Najiar M. Effects of age on validity of self-reported height, weight, and body mass index: findings from the Third National Health and Nutrition Examination Survey, 1988-1994. J Am Diet Assoc 2001:101:28-34; quiz 5-6.

16. Merrill RM, Richardson JS. Validity of self-reported height, weight, and body mas index: findings from the National Health and Nutrition Examination Survey, 2001-2006. Prev Chron Dis 2009;6:A121.

17. Ji CY, Chen TJ. Secular changes in stature and body mass index for Chinese youth in sixteen major cities, 1950s-2005. Am J Hum Biol 2008:20:530-7.

18. Leung SS, Lau JT, Xu YY, et al. Secular changes in standing height, sitting height and sexual maturation of Chinese - the Hong Kong Growth Study, 1993. Ann Hum Biol 1996:23:297-306.

19. Funatogawa I, Funatogawa $\mathrm{T}$, Nakao $\mathrm{M}$, et al. Changes in body mass index by birth cohort in Japanese adults: results from the National Nutrition Survey of Japan 1956-2005. Int J Epidemiol 2009:38:83-92.

20. Hwang JY, Shin C, Frongillo EA, et al. Secular trend in age at menarche for South Korean women born between 1920 and 1986: the Ansan Study. Ann Hum Biol 2003; 30:434-42.

21. Cole TJ. Secular trends in growth. Proc Nutr Soc 2000;59:317-24

22. Silventoinen K. Determinants of variation in adult body height. J Biosoc Sci 2003:35:263-85

23. Gross domestic product (GDP) dollars from 1950 to 2007. Seattle, USA: The Institute for Health Metrics and Evaluation. Univeristy of Washington, WA 98121, 2009

24. Weber G, Seidler $H$, Wilfing $H$, et al. Secular change in height in Austria: an effect of population stratification? Ann Hum Biol 1995:22:277-88.

25. Padez C, Johnston F. Secular trends in male adult height 1904-1996 in relation to place of residence and parent's educational level in Portugal. Ann Hum Biol 1999:26:287-98

26. Kim KB, French KE, Spurgeon JH. Somatic comparisons at four ages of South Korean females and females of other Asian groups. Am J Hum Biol 1999:11:735-44.

27. Luo $\mathbf{Y}$, Yang F, Lei SF, et al. Differences of height and body mass index of youths in urban vs rural areas in Hunan province of China. Ann Hum Biol 2009:1-6.

28. Li L, Dangour $A D$, Power $C$. Early life influences on adult leg and trunk length in the 1958 British birth cohort. Am J Hum Biol 2007;19:836-43.

29. Sorensen HT, Sabroe $\mathrm{S}$, Rothman KJ, et al. Birth weight and length as predictors for adult height. Am J Epidemiol 1999;149:726-9.

30. Webb E, Kuh D, Peasey A, et al. Childhood socioeconomic circumstances and adult height and leg length in central and eastern Europe. J Epidemiol Community Health 2008;62:351-7.

31. Chinn S, Rona RJ, Price CE. The secular trend in height of primary school children in England and Scotland 1972-9 and 1979-86. Ann Hum Biol 1989;16:387-95.

32. Whitley E, Gunnell D, Davey Smith G, et al. Childhood circumstances and anthropometry: the Boyd Orr cohort. Ann Hum Biol 2008;35:518-34.

33. Moyes CD. Stature and birth rank. A study of schoolchildren in St Helena. Arch Dis Child 1981;56:116-20.

34. Hermanussen $\mathbf{M}$, Hermanussen B, Burmeister $\mathrm{J}$. The association between birth order and adult stature. Ann Hum Biol 1988;15:161-5.

35. Ghosh JR, Bandyopadhyay AR. Income, birth order, siblings, and anthropometry. Hum Biol 2006:78:733-41.

36. Aekplakorn W, Mo-Suwan L. Prevalence of obesity in Thailand. Obes Rev 2009:10:589-92.

37. Khuhaprema T, Srivatanakul P, Sriplung $\mathrm{H}$, et al. Cancer in Thailand volume IV 1998-2000. Bangkok: National Cancer Institute, 2007. 